\title{
Nocturnal Blood Pressure Dipping As a Marker of Endothelial Function and Subclinical Atherosclerosis in Pediatric-onset Systemic Lupus Erythematosus
}

\section{Joyce C Chang ( $\nabla$ chang.joyce.c@gmail.com )}

Children's Hospital of Philadelphia https://orcid.org/0000-0002-1691-4814

\section{Rui Xiao}

University of Pennsylvania Perelman School of Medicine

\section{Kevin E Meyers}

Children's Hospital of Philadelphia Division of Nephrology

\section{Laura Mercer-Rosa}

Children's Hospital of Philadelphia Division of Cardiology

\section{Shobha S Natarajan}

Children's Hospital of Philadelphia Division of Cardiology

\section{Pamela F Weiss}

Children's Hospital of Philadelphia Division of Rheumatology

\section{Andrea M Knight}

Hospital for Sick Children

\section{Research article}

Keywords: systemic lupus erythematosus, pediatric systemic lupus erythematosus, ambulatory blood pressure monitoring, cardiovascular disease, cardiovascular diagnostic techniques

Posted Date: May 19th, 2020

DOI: https://doi.org/10.21203/rs.2.19799/v2

License: (c) (i) This work is licensed under a Creative Commons Attribution 4.0 International License. Read Full License

Version of Record: A version of this preprint was published at Arthritis Research \& Therapy on June 3rd, 2020. See the published version at https://doi.org/10.1186/s13075-020-02224-w. 


\section{Abstract}

Background: Loss of the normal nocturnal decline in blood pressure (BP), known as non-dipping, is a potential measure of cardiovascular risk identified by ambulatory blood pressure monitoring (ABPM). We sought to determine whether non-dipping is a useful marker of abnormal vascular function and subclinical atherosclerosis in pediatric-onset systemic lupus erythematosus (pSLE).

Methods: Twenty subjects 9-19 years of age with pSLE underwent ABPM, peripheral endothelial function testing, carotid-femoral pulse wave velocity/analysis for aortic stiffness, and carotid intima-media thickness. We assessed the prevalence of non-dipping and other ABPM abnormalities. Pearson or Spearman rank correlation tests were used to evaluate relationships between nocturnal BP dipping, BP load (\% of abnormally elevated BPs over 24-hours) and vascular outcome measures.

Results: The majority (75\%) of subjects had inactive disease, with mean disease duration of 3.2 years $( \pm 2.1)$. The prevalence of non-dipping was $50 \%$, which occurred even in the absence of nocturnal or daytime hypertension. Reduced diastolic BP dipping was associated with poorer endothelial function ( $\boldsymbol{r}$ $0.5, p=0.04$ ). Intima-media thickness was significantly greater in subjects with non-dipping (mean standard deviation score of 3.0 vs $1.6, p=0.02$ ). In contrast, higher systolic and diastolic BP load were associated with increased aortic stiffness ( $\rho 0.6, \mathrm{p}=0.01$ and $\rho 0.7, \mathrm{p}<0.01$, respectively), but not with endothelial function or intima-media thickness.

Conclusion: In a pSLE cohort with low disease activity, isolated nocturnal BP non-dipping is prevalent and associated with endothelial dysfunction and atherosclerotic changes. In addition to hypertension assessment, ABPM has a promising role in risk stratification and understanding heterogeneous mechanisms of cardiovascular disease in pSLE.

\section{Background}

Premature cardiovascular events remain the major cause of morbidity and mortality among patients with systemic lupus erythematosus (SLE) beyond the first several years of disease,[1-3] and those with pediatric-onset SLE (pSLE) are at risk for cardiovascular events at even younger ages, between early to middle adulthood.[4] Existing non-invasive measures of cardiovascular risk in the pediatric population, such as carotid intima-media thickness (cIMT), have demonstrated accelerated progression of subclinical atherosclerosis in pSLE.[5] However, these methods are highly operator-dependent and primarily limited to research applications. As a result, there is a need for clinically feasible strategies to measure cardiovascular risk in pSLE at early stages when there is an opportunity to intervene.

Loss of nocturnal blood pressure (BP) dipping is a marker of increased cardiovascular risk that can be easily screened for using 24-hour ambulatory blood pressure monitoring (ABPM). Normal blood pressure variability follows a diurnal pattern with a decline in $\mathrm{BP}$ at night. Loss of the physiologic nocturnal $\mathrm{BP}$ decline, also referred to as "non-dipping", is an independent predictor of target organ damage and cardiovascular mortality in both hypertensive and normotensive adults.[6-9] This relationship is 
potentially mediated by endothelial dysfunction, one of the earliest stages of subclinical atherosclerosis (Figure 1).[10] Routine ABPM assessment of non-dipping is recommended in children with juvenile-onset diabetes,[11] as it predicts progression to microalbuminuria.[12] To date, there are no specific guidelines on the use of ABPM in PSLE.

ABPM has several advantages over many research-based non-invasive measures of cardiovascular risk, because it is a clinically available tool with pediatric reference standards and does not suffer from operator-dependence. The magnitude of nocturnal BP dipping is normally distributed, $[13,14]$ with a prevalence of non-dipping of $14 \%$ observed among non-obese children referred for ABPM for elevated casual BP.[15] Despite this, there is very limited data on the use of ABPM in pSLE. Campbell et al. first described ABPM abnormalities in a retrospective study of 10 children with pSLE and identified nondipping in 90\%.[16] However, these ABPM studies were clinically indicated and reflect the prevalence from targeted rather than routine screening. Furthermore, all subjects were taking glucocorticoids at the time of assessment. Canpolat et al. reported a prevalence of non-dipping of $67 \%$ in 24 pSLE subjects in Turkey, the majority of which were also still being treated with glucocorticoids and had active disease at the time of assessment.[17] The prevalence of non-dipping in an unselected U.S. pSLE population and the contribution of glucocorticoid use have not been fully characterized. In addition, the clinical and physiologic relevance of non-dipping to early detection of cardiovascular disease in pSLE remains unknown.

Therefore, the objectives of this study were to: i) estimate the prevalence of nocturnal BP non-dipping in an unselected, racially diverse population of children and adolescents with PSLE, ii) assess convergent validity between nocturnal BP dipping and other non-invasive measures of vascular health, including endothelial dysfunction, arterial stiffness and wall thickening (Figure 1), and iii) determine whether nondipping status can be used to stratify early atherosclerotic risk.

\section{Methods}

Study Design: This was a cross-sectional study of children and adolescents with pSLE who were prospectively recruited to undergo comprehensive non-invasive cardiovascular testing from October 2018 to June 2019.

Study Population: Subjects ages 9-21 years inclusive, meeting American College of Rheumatology or Systemic Lupus International Collaborating Clinics classification criteria for SLE by age 18, were recruited from the pediatric rheumatology clinic at a tertiary academic center. We excluded subjects with chronic kidney disease stage 3 or greater (estimated glomerular filtration rate (eGFR) $<60 \mathrm{~mL} / \mathrm{min} / 1.73 \mathrm{~m}^{2}$ for $\geq 3$ months), on dialysis, after kidney transplantation, or with a history of obstructive sleep apnea, which are known to be independently associated with nocturnal hypertension. We also excluded subjects with known hypertension at enrollment, but patients with a history of resolved hypertension diagnosis were eligible for inclusion. 


\section{Study Measures.}

\section{Blood Pressure Outcomes:}

Casual BP assessment: Trained research nurses measured casual BP using the auscultatory method at least twice in the right upper extremity while in the seated position with an aneroid sphygmomanometer (Mabis MedicKit 5; Mabis Healthcare, Waukegan, IL). If there was a discrepancy of more than $5 \mathrm{mmHg}$ between two measurements in either systolic (SBP) or diastolic blood pressure (DBP), a third measurement was taken and the average of the three measurements was recorded.

Ambulatory BP assessment: 24-hour ABPM was performed using oscillometric Spacelab 90217 monitors (Spacelabs Medical, CA) according to American Heart Association guidelines for standard ambulatory assessment.[18] Systolic and diastolic BP were measured every 20 minutes while awake and every 30 minutes during sleep for 24 hours. The primary outcome was the presence of non-dipping BP, defined as < $10 \%$ decrease from mean daytime to mean nighttime systolic or diastolic BP.[19] Secondary outcomes included masked hypertension (normal casual BP with abnormal mean 24-hr BP or BP load), white coat hypertension (elevated casual BP with normal mean 24-hr BP and BP load), nocturnal hypertension (> $25 \%$ of nighttime BP measurements above the $95^{\text {th }}$ percentile for age, sex and height), and average 24hour systolic or diastolic BP load (total percentage of BP values above the $95^{\text {th }}$ percentile). $[13,14,19] \mathrm{BP}$ percentiles and ABPM hypertension category were classified based on the updated American Academy of Pediatrics clinical practice guidelines.[11]

\section{Measures of Vascular Function and Stiffness:}

Peripheral endothelial function: Endothelial vasomotor function was assessed in the fasting state during morning hours in a temperature controlled vascular lab using the EndoPAT device (Endo-PAT2000, ItamarMedical, Caesarea, Israel), as previously described.[20] Digital pulse amplitude readings pre- and postdeflation were used to calculate the reactive hyperemia index $(\mathrm{InRHI})$, defined as the natural log transformation of the ratio of the post-deflation pulse amplitude over the baseline measurement in the hyperemic finger divided by the corresponding ratio in the control finger. $\mathrm{LnRHI}$ values $\leq 0.51$ are considered abnormal.[21]

Aortic stiffness: Carotid-femoral pulse wave velocity (PWV) was measured using the SphigmoCor Vx system (AtCor Medical Pty Ltd, Australia).[22] PWV standard deviation scores (SDS) for age and sex were calculated using published reference values for mean transit velocity.[23] Pulse wave analysis (PWA) was also performed to calculate the aortic augmentation index, which approximates aortic stiffness.

\section{Structural Measure of Subclinical Atherosclerosis:}

Carotid intima-media thickness (IMT): One experienced sonographer performed all high-resolution, real-time B-mode carotid ultrasound studies (ATL 3000). Serial images in longitudinal and transverse planes were obtained in the supine position at a $45^{\circ}$ angle of insonation. Using edge-tracking software 
(EchoPAC PC, GE Medical Systems), an echocardiologist experienced in measuring carotid IMT (S.N.) performed three separate IMT measurements at the start of the R wave on the electrocardiograph (enddiastole) in the far wall of the right and left distal common carotid artery $10 \mathrm{~mm}$ proximal to the origin of the carotid bifurcation, the carotid bulb, and the internal carotid artery $10 \mathrm{~mm}$ distal to the bifurcation. The mean of the bilateral CCA measurements (CCA-IMT) and the mean of all 6 segments (mean cIMT) were used as the primary outcome measures of intima-media thickness.[24,25] SDS by age and sex for CCA-IMT were calculated by the LMS method using published reference norms.[26]

\section{Covariates:}

Traditional cardiovascular risk factors: Subjects completed height and weight measurements for body mass index (BMI), a Physical Activity Questionnaire,[27] and a demographics survey (race, ethnicity, household income, parental education, family history of early cardiovascular disease). Each subject provided a fasting blood sample for measurement of lipids, high-sensitivity C-reactive protein by immunoassay (Roche Diagnostics c311), and lipoprotein A by enzyme-linked immunosorbent assay (Abcam 212165), which have previously been identified as risk factors for increased rates of atherosclerotic progression in SLE. $[5,28]$ Lipoprotein A values $>95^{\text {th }}$ percentile for age and race/ethnicity (NHANES III) were considered abnormal.[29]

Disease-related factors: Additional clinical data was abstracted from the electronic medical record, including disease duration, SLE manifestations, SLE Disease Activity Index (SLEDAI-2K) scores,[30] glucocorticoid dose, immunosuppressive medication history, and the most recent serum creatinine and urine protein/creatinine levels. eGFR was calculated using the revised Schwartz equation for subjects $£$ 18 years of age and the Chronic Kidney Disease Epidemiology Collaboration equation for subjects $>18$ years of age.[31,32] Cumulative disease activity was calculated as a time-averaged mean [33] using historical abstractions of SLEDAI-2K scores from clinic visits. For subjects who never had hypocomplementemia or anti-dsDNA antibodies as a disease manifestation, the corresponding score component of zero was carried forward. We also retrospectively calculated the proportion of time in a Lupus Low Disease Activity State (LLDAS) to account for glucocorticoid dose.[34]

\section{Statistical Analysis}

ABPM and other vascular outcome measures were summarized using standard descriptive statistics such as mean and standard deviation or median and interquartile range for continuous variables, and count and frequency for categorical variables. We used Shapiro Wilk's test to assess for normality. A prespecified significance level alpha of 0.10 (two-sided) was used for all analyses.

1. To assess convergent validity between the magnitude of nocturnal BP dipping and other measures of vascular health, we used Pearson correlation ( $\boldsymbol{r}$ ) coefficients. Since it is not known whether systolic or diastolic BP is more clinically relevant in SLE, systolic and diastolic BP dip were considered separately. We identified outliers using leverage plots and Cook's distance, and performed sensitivity analyses with and without influential outliers with a Cook's distance $>0.22$. In a secondary 
analysis, we used Spearman rank correlation $(\rho)$ coefficients to evaluate the relationship between 24-hour BP load and other vascular measures.

2. Prevalence of non-dipping and secondary ambulatory BP outcomes was calculated using the total number of adequate ABPM studies as the offset. To identify potential risk factors for non-dipping, differences in clinical characteristics by non-dipping status were assessed using Fisher's exact, Student's t, or Wilcoxon rank sum tests as appropriate.

3. To determine whether non-dipping is associated with atherosclerotic risk, we used two-sample t-tests to test differences in CCA-IMT and mean cIMT between subjects with normal nocturnal dipping and those with non-dipping. To explore the discriminative ability of non-dipping with respect to high risk CCA-IMT (SDS > 2.0), we assessed concordance using the C-statistic.

\section{Results}

The mean age was 16.5 years (range $9-19)$ and the average disease duration was 3.2 years $( \pm 2.1)$ (Table 1). $40 \%$ of subjects were African American. $75 \%$ of subjects had inactive disease (SLEDAI-2K score $<4$ ) at the time of enrollment. $25 \%$ of subjects had a history of nephritis, of which only one had ongoing proteinuria and all had normal renal function (eGFR $>90 \mathrm{~mL} / \mathrm{min} / 1.73 \mathrm{~m}^{2}$ ).

ABPM was evaluable and well-tolerated in $18 / 20$ subjects. Of the two subjects with insufficient ABPM data, one did not wish to be seen wearing the monitor at school, while the other was intolerant of cuff inflations. EndoPAT interpretation was precluded in two subjects, one due to vasculitic finger lesions resulting in poor waveforms, and another due to pre-pubertal age with small finger size, resulting in falsely low amplitudes. No subjects had active Raynaud's at the time of assessment.

\section{Prevalence of Non-Dipping and Other Ambulatory BP Abnormalities}

The prevalence of non-dipping was $50 \%$, which commonly occurred in the setting of otherwise normal ambulatory BP (Table 2). Two subjects (11\%) had nocturnal hypertension, one of which also had nondipping. All subjects who met criteria for non-dipping had reduced SBP dip, one of which also had reduced DBP dip. On average, the magnitude of nocturnal DBP dipping was greater (mean $17.6 \%$, SD 5.7) compared to SBP dipping (mean 10.0\%, SD 3.7). Agreement between hypertension class by casual BP measurement and ambulatory BP was poor (observed agreement $56 \%$ ), with 6 cases of white coat hypertension and one case of masked hypertension (Table 2). There were no significant differences in 24hour BP load among non-dippers compared to those with normal BP dipping (mean SBP load $4 \%$ vs. $12 \%$, $p=0.18$; mean DBP load $8 \%$ vs. $11 \%, p=0.96$ ). There was also no association between the magnitude of SBP dipping and nocturnal SBP load $(\rho=0.04, \mathrm{p}=0.88)$ or DBP dipping and nocturnal DBP load $(\rho=-0.29$, $p=0.24)$.

Patient Characteristics Associated with Non-Dipping BP Pattern 
Subjects with non-dipping BP had higher mean BMI (mean $81^{\text {st }}$ percentile vs. $56^{\text {th }}$ percentile, $p=0.05$ ) and greater high-density lipoprotein levels (mean $59( \pm 8)$ vs. $44( \pm 5) \mathrm{mg} / \mathrm{dL}, \mathrm{p}<0.01$ ) compared to subjects with normal BP dipping (Table 1). $44 \%$ of non-dippers were still using glucocorticoids compared to $11 \%$ of subjects with normal dipping $(p=0.29)$. The remaining $56 \%$ of non-dippers had discontinued all glucocorticoids a median of 367 days (range 308-1367) before the study visit. The mean timeaveraged cumulative disease activity was $6.3( \pm 4.6)$ among non-dippers compared to $3.9( \pm 2.1)$ among subjects with normal dipping, although the difference was not statistically significant $(p=0.14)$.

\section{Association Between Nocturnal BP Dipping and Vascular Function and Stiffness}

Peripheral endothelial function. The prevalence of endothelial dysfunction was $22 \%$ (Table 3). There was a significant association between greater magnitudes of DBP dipping and better endothelial function ( $\boldsymbol{r}$ $0.5, p$-value $0.04, n=18$ ). In contrast, BP load did not correlate with endothelial function (Table 4).

Aortic stiffness. Abnormal PWV (SDS > 2) was observed in only one subject with an inadequate ABPM study. Mean augmentation index was $0.9( \pm 13.7)$. Higher estimates of aortic stiffness by augmentation index, but not PWV, were strongly correlated with both 24-hour SBP and DBP load ( $\rho 0.6$ and 0.7, respectively, $p$-values $£ 0.01$ ). In contrast to endothelial function, there was no significant correlation between SBP/DBP dipping and measures of aortic stiffness (Table 4).

\section{Non-dipping as a Marker of Subclinical Atherosclerosis}

Carotid intima-media thickness. The average CCA-IMT SDS was 2.36 standard deviations above the norm for age and sex (Table 3). Non-dipping BP pattern was associated with statistically significant increases in IMT across all carotid segments evaluated (Table 5). This difference was primarily driven by changes in systolic dip. A lower magnitude of SBP dipping correlated with significantly greater CCA-IMT ( $\boldsymbol{r}-0.47$, p-value 0.06) and ICA-IMT ( $\boldsymbol{r}-0.58$, p-value 0.03) (Table 4). In contrast, there was no significant correlation between the magnitude of DBP dipping and CCA-IMT ( $\boldsymbol{r}-0.17$, p-value 0.49). There were also no significant associations between SBP or DBP load and IMT outcome measures (Supplemental Table).

CCA-IMT was abnormally increased (SDS > 2.0) in $60 \%$ of the total cohort and $56 \%$ of those with adequate ABPM studies. Non-dipping BP was observed in 7/10 subjects with increased CCA-IMT SDS compared to $2 / 8$ subjects with normal CCA-IMT SDS, corresponding to a C-statistic of $72 \%$ of subjects correctly classified.

\section{Discussion}

In this study of comprehensive pSLE cardiovascular profiles, there are several important findings that inform the utility and interpretation of ABPM in pSLE. First, systolic non-dipping was significantly associated with increased carotid IMT, which supports the potential role for ABPM in assessment of atherosclerotic risk in this population. Second, decreased diastolic BP dipping was associated with poorer endothelial function, suggesting that ABPM may reveal information about heterogenous mechanisms of 
cardiovascular risk in pSLE. Lastly, non-dipping BP patterns were common in this cohort of pSLE subjects despite low disease activity, and these findings were independent of hypertension and glucocorticoid use. This study supports the potential value of utilizing ABPM to assess cardiovascular health in this high-risk population.

In support of the relevance of isolated non-dipping to cardiovascular risk in PSLE, we observed that loss of systolic BP dipping was the single ABPM measurement that was most strongly associated with structural evidence of subclinical atherosclerosis by $\mathrm{CIMT}$. This suggests that there may be important clinical implications of non-dipping even in normotensive children and adolescents with pSLE. In addition, non-dipping may be particularly useful as a screening tool in pSLE because it is clinically feasible and less operator-dependent than cIMT. Higher frequencies of non-dipping (31-40\%) have also been observed in pediatric obesity.[15,35] Although obesity may contribute to the relationship between non-dipping and increased cIMT in our study,[36,37] a similar prevalence of non-dipping and association with cIMT was reported in a Turkish cohort of pSLE patients who had normal BMI,[17] and therefore obesity is unlikely the only explanation for our findings. Larger ABPM studies will be needed to distinguish direct and indirect effects of disease-specific factors and potential confounders such as obesity. In addition, in this primarily normotensive cohort of pSLE subjects, non-dipping was a better predictor of increased cIMT than 24 -hr blood pressure or BP load. Further investigation is warranted to determine whether non-dipping in pSLE patients is independently prognostic of worse cardiovascular outcomes.

In addition to structural changes of subclinical atherosclerosis, nocturnal BP dipping patterns may also provide information about endothelial function. In our study, lower magnitudes of diastolic BP dipping correlated with worse peripheral endothelial function by digital reactive hyperemia. Associations between non-dipping and endothelial function have also been observed in pediatric hematopoietic cell transplant patients[38] and adults with resistant hypertension.[39] Furthermore, abnormal digital reactive hyperemia by EndoPAT has been shown to be predictive of cardiovascular events in adults,[40] especially nonobstructive coronary events in women mediated by coronary microvascular dysfunction.[41] Therefore, the lack of concurrent association between diastolic BP dipping and cIMT (as initially hypothesized in Figure 1) could potentially be explained by a more direct pathway from endothelial dysfunction to nonobstructive coronary disease, independent of atherosclerotic burden. Endothelial dysfunction has previously been proposed as a pathophysiologic mechanism of the association between non-dipping and cardiovascular disease.[42-44] Studies in adults with SLE suggest that high IFNa expression impairs nitric oxide release, which promotes endothelial dysfunction and supports the biologic plausibility of this mechanism of cardiovascular risk in SLE. $[45,46]$ More importantly, both endothelial function and the cardiovascular risk associated with non-dipping have been shown to be modifiable in other conditions, including rheumatoid arthritis and hypertension, respectively, $[47,48]$ demonstrating reversibility of these measures and potential opportunities for intervention in SLE. This highlights the need for further studies of the longitudinal relationships between non-dipping and endothelial function in PSLE with cardiovascular outcomes such as non-obstructive coronary artery disease. 
Our study also provides evidence that secondary risk factors such as hypertension, renal disease and glucocorticoid use are not sufficient to explain the high prevalence of non-dipping in pSLE. Nondipping was frequently an isolated finding and independent of both systolic and diastolic BP load. This suggests that in chronic inflammatory states such as pSLE, non-dipping is less likely to be on a simple continuum with nocturnal or daytime hypertension. Chronic kidney disease is also a known independent risk factor for non-dipping,[49] however the majority of non-dippers in our study either never had renal involvement or had inactive nephritis with normal renal function. Therefore, ABPM may have an important role in pSLE even in the absence of known renal involvement. Disruptions in diurnal BP patterns are hypothesized to result from dysregulation of neurohumoral regulatory pathways that follow circadian rhythms, providing qualitatively different information than overall elevations in BP.[50] Glucocorticoids may blunt diurnal variation by disrupting the hypothalamic-pituitary adrenal (HPA) axis. However, the majority of pSLE subjects with non-dipping in our study had discontinued glucocorticoids a year or more prior, and therefore disruption of the HPA axis by glucocorticoids is also insufficient to explain the prevalence of non-dipping in PSLE.

Lastly, while further investigation is required to determine the role of nocturnal BP dipping for cardiovascular health assessment in PSLE, ABPM has clear utility for hypertension assessment. The agreement between ambulatory and casual BP measurements in this study was extremely poor, with one case of masked hypertension resulting in initiation of antihypertensive treatment, as well as 6 cases of white coat hypertension. Of note, all of the study visits started in the early morning, so the discrepancy between ambulatory and casual BP could have been inflated by morning blood pressure surge,[51] which has yet to be studied in children. Measurement of ambulatory blood pressure in pSLE provides a more accurate assessment of cardiovascular risk related to hypertension and should be considered for routine screening in addition to office-based casual BP measurement.

Strengths of this study include the use of multimodality vascular assessments protocolized for research purposes, which contributes to our understanding of the physiologic relevance of non-dipping. This is the first study to assess peripheral endothelial function by digital reactive hyperemia in pSLE, which is well tolerated and more easily reproducible than operator-dependent methods such as brachial artery reactivity by flow-mediated dilation.[52] In addition, the racial, ethnic and socioeconomic diversity of this study cohort contribute to the generalizability of our findings. There are also several limitations. Due to the small sample size and cross-sectional design, we were unable to perform multivariable adjustments or assess for effect modification. In addition, although 24-hr ABPM was well-tolerated in most cases, we did not directly assess sleep quality, and therefore the impact of sleep on ABPM measurements will need to be accounted for in future studies. With respect to EndoPAT technology, readings are not interpretable in pSLE patients with active Raynaud's or digital vasculitis. Falsely low readings have also been reported in young children due to the one size fit all probe,[52] and therefore separate validation studies would need to be conducted before application of this method in rheumatic conditions that affect pre-pubertal children. 


\section{Conclusions}

In summary, loss of nocturnal BP dipping is common even in PSLE patients without clinically suspected hypertension, and may serve as a useful surrogate marker of early vascular dysfunction and subclinical atherosclerosis. Our findings suggest that attenuation of either systolic or diastolic BP dipping may be indicative of different pathologic cardiovascular states in PSLE. With the added utility for hypertension assessment, ABPM could potentially be used to screen for multiple mechanisms of cardiovascular risk. Larger longitudinal studies are warranted to determine whether non-dipping is predictive of vascular disease progression, and whether it may serve as a potential therapeutic target in pSLE. In addition, further investigation of the pathways through which pSLE and its comorbidities influence ABPM measures is needed to inform the development of guidelines for ABPM use and interpretation in pSLE.

\section{List Of Abbreviations}

BP - blood pressure; pSLE - pediatric-onset systemic lupus erythematosus; ABPM - ambulatory blood pressure monitoring; CIMT - carotid intima-media thickness; SBP - systolic blood pressure; DBP diastolic blood pressure; InRHI - natural log of the reactive hyperemia index; PWV - pulse wave velocity; SDS - standard deviation score; PWA - pulse wave analysis; CCA - common carotid artery; ICA internal carotid artery; BMI - body mass index; SLEDAI-2K - systemic lupus erythematosus disease activity index; LLDAS - lupus low disease activity state

\section{Declarations}

Ethics approval and consent to participate: The Children's Hospital of Philadelphia Institutional Review Board approved this study (\#18-015264). Written informed consent was obtained for all subjects from parents/legal guardians or patients age 18 and over, and child assent was obtained for subjects under age 18.

Consent for publication: Not applicable

Availability of data and materials: The data that support the findings of this study are available on request from the corresponding author (J.C.). The data are not publicly available due to information that could compromise research participant privacy.

Competing interests: The authors declare that they have no competing interests.

Funding: This work was supported by the Lupus Foundation of America, Inc. Gary S. Gilkeson Career Development Award (J.C.) and by Grant Number UL1TR001878 from the National Center for Advancing Translational Sciences, National Institutes of Health. Support for L.M. from NIH K01HL125521 and the Pulmonary Hypertension Association Supplement to K01HL125521. The content is solely the responsibility of the authors and does not necessarily represent the official views of the LFA or the NIH. 
Authors' contributions: J.C. designed the study, collected and analyzed the data, and drafted the manuscript. R.X. contributed to data analysis and substantially revised the manuscript. K.M contributed to the study design, acquisition and interpretation of ambulatory blood pressure monitoring data; S.N. contributed to carotid ultrasound data acquisition and interpretation, and substantially revised the manuscript; L.M. contributed to the study design and interpretation of cardiovascular data, and substantially revised the manuscript; P.W. contributed to the conception and design of the study, data analysis, and substantially revised the manuscript. A.K. contributed to the conception and design of the study, data analysis, and substantially revised the manuscript. All authors read and approved the final manuscript.

Acknowledgments: CHOP Echolab Research Unit: Craig Tinker performed all carotid ultrasound and tonometric studies. Anysia Fedec performed tissue Doppler measurements for echocardiograms. CHOP Rheumatology Research Core: Taylor Goldberg and Sarah McGuire assisted with study coordination and chart abstractions, and Lindsay N. Waqar, MPH supervised study coordination.

\section{References}

1. Hak AE, Karlson EW, Feskanich D, Stampfer MJ, Costenbader KH. Systemic lupus erythematosus and the risk of cardiovascular disease: Results from the nurses' health study. Arthritis Rheum. 2009;61:1396-402.

2. Björnådal L, Yin L, Granath F, Klareskog L, Ekbom A. Cardiovascular disease a hazard despite improved prognosis in patients with systemic lupus erythematosus: results from a Swedish population based study 1964-95. J Rheumatol. 2004;31:713-9.

3. Hanly JG, Li Q, Su L, Urowitz MB, Gordon C, Bae S-C, et al. Cerebrovascular Events in Systemic Lupus Erythematosus. Arthritis Care Res. 2018;70:1478-87.

4. Hersh AO, Trupin L, Yazdany J, Panopalis P, Julian L, Katz P, et al. Childhood-onset disease as a predictor of mortality in an adult cohort of patients with systemic lupus erythematosus. Arthritis Care Res. 2010;62:1152-9.

5. Schanberg LE, Sandborg C, Barnhart HX, Ardoin SP, Yow E, Evans GW, et al. Premature atherosclerosis in pediatric systemic lupus erythematosus: risk factors for increased carotid intimamedia thickness in the atherosclerosis prevention in pediatric lupus erythematosus cohort. Arthritis Rheum. 2009;60:1496-507.

6. Ohkubo T, Hozawa A, Yamaguchi J, Kikuya M, Ohmori K, Michimata M, et al. Prognostic significance of the nocturnal decline in blood pressure in individuals with and without high 24-h blood pressure: the Ohasama study. J Hypertens. 2002;20:2183-9.

7. Salles GF, Reboldi G, Fagard RH, Cardoso CRL, Pierdomenico SD, Verdecchia P, et al. Prognostic Effect of the Nocturnal Blood Pressure Fall in Hypertensive Patients The Ambulatory Blood Pressure Collaboration in Patients With Hypertension (ABC-H) Meta-Analysis. Hypertension. 2016;67:693-700. 
8. Fagard RH, Celis H, Thijs L, Staessen JA, Clement DL, Buyzere MLD, et al. Daytime and Nighttime Blood Pressure as Predictors of Death and Cause-Specific Cardiovascular Events in Hypertension. Hypertension. 2008;51:55-61.

9. Hoshide S. Associations between nondipping of nocturnal blood pressure decrease and cardiovascular target organ damage in strictly selected community-dwelling normotensives. Am J Hypertens. 2003;16:434-8.

10. Steyers C, Miller F. Endothelial Dysfunction in Chronic Inflammatory Diseases. Int J Mol Sci. 2014;15:11324-49.

11. Flynn JT, Kaelber DC, Baker-Smith CM, Blowey D, Carroll AE, Daniels SR, et al. Clinical Practice Guideline for Screening and Management of High Blood Pressure in Children and Adolescents. Pediatrics. 2017;140:e20171904.

12. Lurbe E, Redon J, Kesani A, Pascual JM, Tacons J, Alvarez V, et al. Increase in Nocturnal Blood Pressure and Progression to Microalbuminuria in Type 1 Diabetes. N Engl J Med. 2002;347:797-805.

13. Soergel M, Kirschstein M, Busch C, Danne T, Gellermann J, Holl R, et al. Oscillometric twenty-fourhour ambulatory blood pressure values in healthy children and adolescents: a multicenter trial including 1141 subjects. J Pediatr. 1997;130:178-84.

14. Wühl E, Witte K, Soergel M, Mehls O, Schaefer F, German Working Group on Pediatric Hypertension. Distribution of 24-h ambulatory blood pressure in children: normalized reference values and role of body dimensions. J Hypertens. 2002;20:1995-2007.

15. Macumber IR, Weiss NS, Halbach SM, Hanevold CD, Flynn JT. The Association of Pediatric Obesity With Nocturnal Non-Dipping on 24-Hour Ambulatory Blood Pressure Monitoring. Am J Hypertens. 2016;29:647-52.

16. Campbell JF, Swartz SJ, Wenderfer SE. Nocturnal Hypertension and Attenuated Nocturnal Blood Pressure Dipping is Common in Pediatric Lupus. F1000Research. 2015;4:164.

17. Canpolat N, Kasapcopur O, Caliskan S, Gokalp S, Bor M, Tasdemir M, et al. Ambulatory blood pressure and subclinical cardiovascular disease in patients with juvenile-onset systemic lupus erythematosus. Pediatr Nephrol. 2013;28:305-13.

18. Flynn JT, Daniels SR, Hayman LL, Maahs DM, McCrindle BW, Mitsnefes M, et al. Update: Ambulatory Blood Pressure Monitoring in Children and Adolescents: A Scientific Statement From the American Heart Association. Hypertension. 2014;63:1116-35.

19. Urbina E, Alpert B, Flynn J, Hayman L, Harshfield GA, Jacobson M, et al. Ambulatory blood pressure monitoring in children and adolescents: recommendations for standard assessment: a scientific statement from the American Heart Association Atherosclerosis, Hypertension, and Obesity in Youth Committee of the council on cardiovascular disease in the young and the council for high blood pressure research. Hypertension. 2008;52:433-51.

20. Kuvin JT, Patel AR, Sliney KA, Pandian NG, Sheffy J, Schnall RP, et al. Assessment of peripheral vascular endothelial function with finger arterial pulse wave amplitude. Am Heart J. 2003;146:16874. 
21. Itamar Medical [Internet]. Itamar Med. [cited 2019 Oct 18]. Available from: http://www.itamarmedical.com/pat_technology/

22. Thurn D, Doyon A, Sözeri B, Bayazit AK, Canpolat N, Duzova A, et al. Aortic Pulse Wave Velocity in Healthy Children and Adolescents: Reference Values for the Vicorder Device and Modifying Factors. Am J Hypertens. 2015;28:1480-8.

23. Reusz GS, Cseprekal O, Temmar M, Kis É, Cherif AB, Thaleb A, et al. Reference Values of Pulse Wave Velocity in Healthy Children and Teenagers. Hypertension. 2010;56:217-24.

24. Dalla Pozza R, Ehringer-Schetitska D, Fritsch P, Jokinen E, Petropoulos A, Oberhoffer R, et al. Intima media thickness measurement in children: A statement from the Association for European Paediatric Cardiology (AEPC) Working Group on Cardiovascular Prevention endorsed by the Association for European Paediatric Cardiology. Atherosclerosis. 2015;238:380-7.

25. Wiegman A, Hutten BA, de Groot E, Rodenburg J, Bakker HD, Büller HR, et al. Efficacy and safety of statin therapy in children with familial hypercholesterolemia: a randomized controlled trial. JAMA. 2004;292:331-7.

26. Doyon A, Kracht D, Bayazit AK, Deveci M, Duzova A, Krmar RT, et al. Carotid artery intima-media thickness and distensibility in children and adolescents: reference values and role of body dimensions. Hypertension. 2013;62:550-6.

27. Kowalski KC, Crocker PRE, Faulkner RA. Validation of the Physical Activity Questionnaire for Older Children. Pediatr Exerc Sci. 1997;9:174-86.

28. Lertratanakul A, Wu P, Dyer AR, Kondos G, Edmundowicz D, Carr J, et al. Risk Factors in the Progression of Subclinical Atherosclerosis in Women With Systemic Lupus Erythematosus: Progression of Subclinical Atherosclerosis in Women With SLE. Arthritis Care Res. 2014;66:1177-85.

29. Obisesan TO, Aliyu MH, Adediran AS, Bond V, Maxwell CJ, Rotimi CN. Correlates of serum lipoprotein (A) in children and adolescents in the United States. The third National Health Nutrition and Examination Survey (NHANES-III). Lipids Health Dis. 2004;3:29.

30. Bombardier C, Gladman DD, Urowitz MB, Caron D, Chang CH. Derivation of the SLEDAI. A disease activity index for lupus patients. The Committee on Prognosis Studies in SLE. Arthritis Rheum. 1992;35:630-40.

31. Schwartz GJ, Muñoz A, Schneider MF, Mak RH, Kaskel F, Warady BA, et al. New equations to estimate GFR in children with CKD. J Am Soc Nephrol JASN. 2009;20:629-37.

32. Levey AS, Stevens LA, Schmid CH, Zhang YL, Castro AF, Feldman HI, et al. A new equation to estimate glomerular filtration rate. Ann Intern Med. 2009;150:604-12.

33. Nikpour M, Urowitz MB, Ibanez D, Harvey PJ, Gladman DD. Importance of cumulative exposure to elevated cholesterol and blood pressure in development of atherosclerotic coronary artery disease in systemic lupus erythematosus: a prospective proof-of-concept cohort study. Arthritis Res Ther. 2011;13:R156.

34. Franklyn K, Lau CS, Navarra SV, Louthrenoo W, Lateef A, Hamijoyo L, et al. Definition and initial validation of a Lupus Low Disease Activity State (LLDAS). Ann Rheum Dis. 2016;75:1615-21. 
35. Westerståhl M, Hedvall Kallerman P, Hagman E, Ek AE, Rössner SM, Marcus C. Nocturnal blood pressure non-dipping is prevalent in severely obese, prepubertal and early pubertal children. Acta Paediatr. 2014;103:225-30.

36. Jourdan C, Wühl E, Litwin M, Fahr K, Trelewicz J, Jobs K, et al. Normative values for intima-media thickness and distensibility of large arteries in healthy adolescents. J Hypertens. 2005;23:1707-15.

37. Lande MB, Carson NL, Roy J, Meagher CC. Effects of Childhood Primary Hypertension on Carotid Intima Media Thickness: A Matched Controlled Study. Hypertension. 2006;48:40-4.

38. Pao E, Gove NE, Flynn JT, Hingorani S. Ambulatory Blood Pressure and Endothelial Dysfunction in Hematopoietic Cell Transplantation Recipients. Biol Blood Marrow Transplant. 2018;24:1678-84.

39. Quinaglia T, Martins LC, Figueiredo VN, Santos RC, Yugar-Toledo JC, Martin JFV, et al. Non-dipping pattern relates to endothelial dysfunction in patients with uncontrolled resistant hypertension. $J$ Hum Hypertens. 2011;25:656-64.

40. Matsuzawa Y, Kwon T-G, Lennon RJ, Lerman LO, Lerman A. Prognostic Value of Flow-Mediated Vasodilation in Brachial Artery and Fingertip Artery for Cardiovascular Events: A Systematic Review and Meta-Analysis. J Am Heart Assoc. 2015;4:e002270-e002270.

41. Matsuzawa Y, Sugiyama S, Sugamura K, Nozaki T, Ohba K, Konishi M, et al. Digital Assessment of Endothelial Function and Ischemic Heart Disease in Women. J Am Coll Cardiol. 2010;55:1688-96.

42. Higashi Y, Nakagawa K, Kimura M, Noma K, Hara K, Sasaki S, et al. Circadian variation of blood pressure and endothelial function in patients with essential hypertension: a comparison of dippers and non-dippers. J Am Coll Cardiol. 2002;40:2039-43.

43. Fontes-Guerra PCA, Cardoso CRL, Muxfeldt ES, Salles GF. Nitroglycerin-mediated, but not flowmediated vasodilation, is associated with blunted nocturnal blood pressure fall in patients with resistant hypertension: J Hypertens. 2015;33:1666-75.

44. von Kanel R, Jain S, Mills PJ, Nelesen RA, Adler KA, Hong S, et al. Relation of nocturnal blood pressure dipping to cellular adhesion, inflammation and hemostasis: J Hypertens. 2004;22:2087-93.

45. Buie JJ, Renaud LL, Muise-Helmericks R, Oates JC. IFN-a Negatively Regulates the Expression of Endothelial Nitric Oxide Synthase and Nitric Oxide Production: Implications for Systemic Lupus Erythematosus. J Immunol. 2017;199:1979-88.

46. Iriyoda TMV, Stadtlober N, Lozovoy M a. B, Delongui F, Costa NT, Reiche EMV, et al. Reduction of nitric oxide and DNA/RNA oxidation products are associated with active disease in systemic lupus erythematosus patients. Lupus. 2017;26:1106-11.

47. Ikonomidis I, Tzortzis S, Lekakis J, Paraskevaidis I, Andreadou I, Nikolaou M, et al. Lowering interleukin-1 activity with anakinra improves myocardial deformation in rheumatoid arthritis. Heart. 2009;95:1502-7.

48. Hermida RC, Ayala DE, Mojón A, Fernández JR. Decreasing Sleep-Time Blood Pressure Determined by Ambulatory Monitoring Reduces Cardiovascular Risk. J Am Coll Cardiol. 2011;58:1165-73.

49. Fedecostante M, Spannella F, Cola G, Espinosa E, Dessì-Fulgheri P, Sarzani R. Chronic Kidney Disease Is Characterized by "Double Trouble" Higher Pulse Pressure plus Night-Time Systolic Blood Pressure 
and More Severe Cardiac Damage. Mukhopadhyay P, editor. PLoS ONE. 2014;9:e86155.

50. Grassi G, Bombelli M, Seravalle G, Dell'Oro R, Quarti-Trevano F. Diurnal blood pressure variation and sympathetic activity. Hypertens Res. 2010;33:381-5.

51. Kario Kazuomi. Morning Surge in Blood Pressure and Cardiovascular Risk. Hypertension. 2010;56:765-73.

52. Selamet Tierney ES, Newburger JW, Gauvreau K, Geva J, Coogan E, Colan SD, et al. Endothelial Pulse Amplitude Testing: Feasibility and Reproducibility in Adolescents. J Pediatr. 2009;154:901-5.

\section{Tables}


Table 1. Clinical characteristics of pSLE subjects by nocturnal BP dipping status

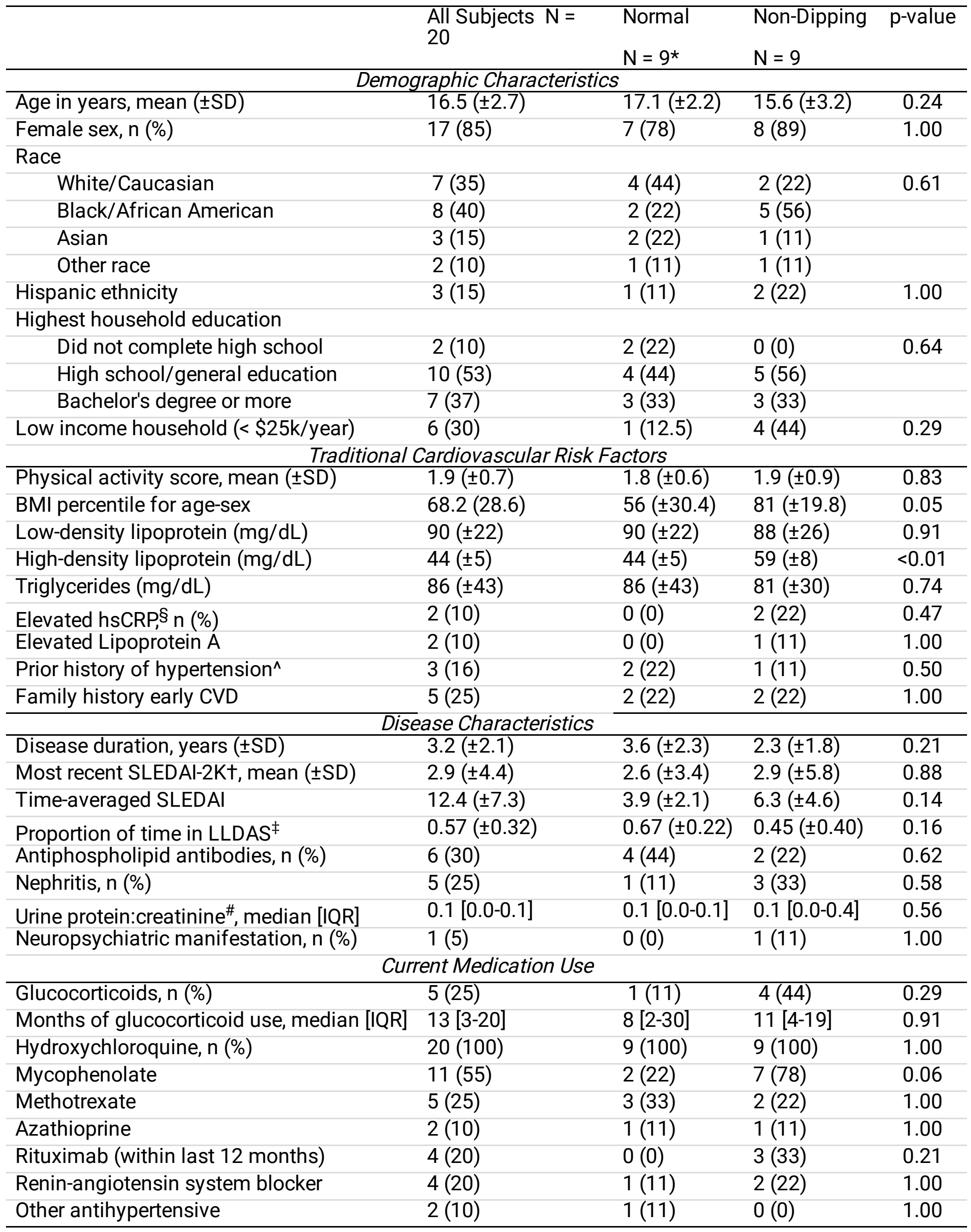


Comparison of baseline clinical characteristics by non-dipping status using Fisher's exact, Student's t-test or Wilcoxon ranksum test as appropriate

* Only 18/20 subjects completed ABPM wear to determine normal dipping vs non-dipping

${ }^{\wedge}$ Previous hypertension diagnosis resolved by physician prior to enrollment

$\S$ High-sensitivity C-reactive protein $>3.0 \mathrm{mg} / \mathrm{L}$

† SLEDAI < 5: low disease activity; 6-10: moderate; 11-19 high; maximum 105

¥ Lupus Low Disease Activity State

\# Random (spot) urine protein to creatinine ratio

Table 2. Presence of non-dipping BP in pSLE despite otherwise normal ABPM

\begin{tabular}{lcc}
\hline & Adequate ABPM studies & Non-dipping BP \\
\hline Normal, $\mathrm{n}(\%)$ & $\mathrm{N}=18$ & $\mathrm{~N}=9$ \\
\hline White coat hypertension & $10(55 \%)$ & $5(56 \%)$ \\
Masked hypertension & $6(33 \%)$ & $3(33 \%)$ \\
\hline Unclassified* & $1(6 \%)$ & $0(0 \%)$ \\
\hline
\end{tabular}

Blood pressure categories based on updated definitions from the 2017 AAP guidelines:

Nocturnal Hypertension: SBP/DBP load $\geq 50 \%$ by ABPM

White coat hypertension: Casual BP $\geq 95^{\text {th }}$ percentile but normal ABPM (mean 24 -hr SBP/DBP $<95^{\text {th }}$ percentile and SBP/DBP load $<25 \%$ )

Masked hypertension: normal clinic BP but mean 24-hr SBP/DBP $>95^{\text {th }}$ percentile and SBP/DBP load $\geq 25 \%$ by ABPM

*Unclassified: elevated clinic BP $\left(>90^{\text {th }}\right.$ but $<95^{\text {th }}$ percentile) with normal ABPM

Table 3. Summary of vascular outcome measures in pSLE subjects

\begin{tabular}{|c|c|c|c|c|}
\hline & & & & Abnormal \\
\hline & $\mathrm{n}$ & mean & SD & $\mathrm{n}(\%)$ \\
\hline $\operatorname{lnRHI}{ }^{*}$ & 18 & 0.74 & 0.24 & $4(22 \%)$ \\
\hline $\mathrm{PWV}, \mathrm{m} / \mathrm{s}$ & 20 & 4.81 & 0.93 & \\
\hline PWV SDS for age/sex & 20 & -0.75 & 1.29 & $1(5 \%)$ \\
\hline Augmentation index, \% & 20 & 0.9 & 13.7 & \\
\hline CCA-IMT, mm & 20 & 0.500 & 0.056 & \\
\hline CCA-IMT SDS for age/sex ${ }^{\dagger}$ & 20 & 2.36 & 1.26 & $12(60 \%)$ \\
\hline
\end{tabular}

* Reactive hyperemia index; InRHI $\leq 0.51$ was considered abnormal; waveforms were uninterpretable in 2 subjects

‡ Pulse wave velocity standard deviation scores for age and sex derived from published reference norms; SDS $>2.0$ was considered abnormal

† Common carotid intima-media thickness standard deviation scores derived from published references norms; SDS > 2.0 was

considered abnormal 
Table 4. Magnitude of nocturnal BP dipping correlates with vascular function and subclinical atherosclerosis

\begin{tabular}{|c|c|c|c|c|c|c|c|c|c|}
\hline \multirow[b]{2}{*}{ Measure } & \multirow[b]{2}{*}{$\mathrm{N}$} & \multicolumn{2}{|c|}{ \% SBP Dip } & \multicolumn{2}{|c|}{ \% DBP Dip } & \multicolumn{2}{|c|}{ SBP load } & \multicolumn{2}{|c|}{ DBP load } \\
\hline & & $\boldsymbol{r}$ & $p$-value & $\boldsymbol{r}$ & $p$-value & $\rho$ & p-value & $\rho$ & p-value \\
\hline \multicolumn{10}{|l|}{ Aortic stiffness } \\
\hline PWV SDS & 18 & 0.3 & 0.29 & -0.1 & 0.76 & 0.3 & 0.28 & 0.0 & 0.87 \\
\hline Augmentation index & 18 & 0.3 & 0.30 & 0.3 & 0.31 & 0.6 & 0.01 & 0.7 & $<0.01$ \\
\hline \multicolumn{10}{|l|}{ Endothelial function } \\
\hline InRHI & 16 & 0.2 & 0.49 & 0.5 & 0.04 & -0.2 & 0.50 & -0.2 & 0.44 \\
\hline \multicolumn{10}{|l|}{ Wall thickening } \\
\hline CCA-IMT & 18 & -0.5 & 0.06 & -0.2 & 0.49 & -0.1 & 0.62 & -0.3 & 0.27 \\
\hline Mean clMT & 14 & -0.6 & 0.03 & -0.1 & 0.72 & -0.2 & 0.51 & -0.1 & 0.79 \\
\hline
\end{tabular}

Pearson's correlation coefficients (r) estimating the relationship between the percent dip in nocturnal BP and measures of vascular function and subclinical atherosclerosis; Spearman rank correlations $(\rho)$ were used to assess associations with 24hour BP load.

SBP = systolic blood pressure; DBP = diastolic blood pressure; PWV SDS = pulse wave velocity standard deviation score for age and sex; $\mathrm{InRHI}=$ reactive hyperemia index; CCA-IMT = mean common carotid intima-media thickness; Mean cIMT = mean of IMT measurements over 6 carotid sites

Table 5. Intima-media thickness is greater in pSLE subjects with non-dipping BP

\begin{tabular}{lccc}
\hline & Normal & Non-dipping & \\
\hline Common carotid (CCA-IMT) & $\mathrm{N}=9$ & $\mathrm{~N}=9$ & p-value* $^{\star}$ \\
\multicolumn{1}{c}{ SDS for age/sext } & $0.466(0.048)$ & $0.525(0.049)$ & 0.02 \\
\hline Carotid bulb-IMT & $1.6(1.1)$ & $3.0(1.2)$ & 0.015 \\
\hline Internal carotid (ICA-IMT)^ & $0.438(0.061)$ & $0.511(0.080)$ & 0.047 \\
\hline Mean clMT (6 sites) & $0.387(0.039)$ & $0.472(0.081)$ & 0.023 \\
\hline
\end{tabular}

*Comparison of absolute cIMT $(\mathrm{mm})$ and standard deviation scores (SDS) by non-dipping status using Student's t-test

† Standard deviation scores for age and sex derived from published references norms (Doyon et al.)

${ }^{\wedge}$ Only $n=14$ evaluable studies

Figures 


\section{Vascular Injury Functional Change Structural Remodeling}

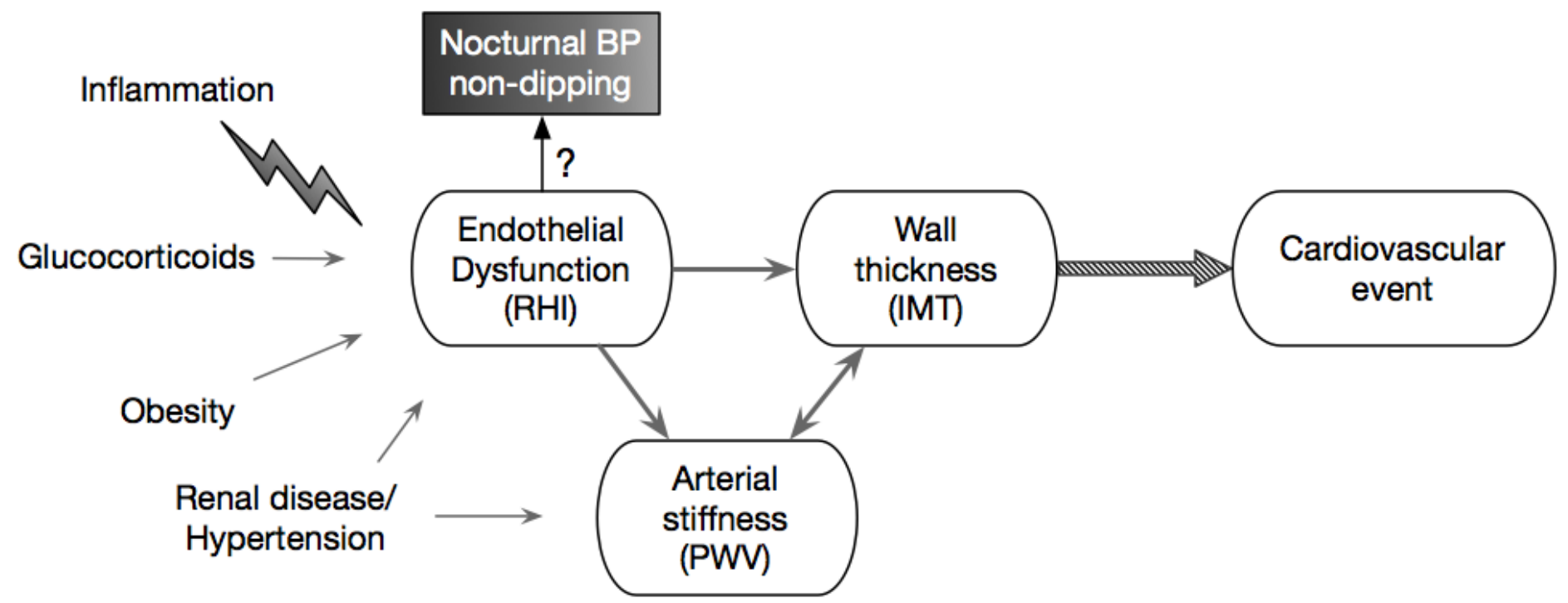

\section{Figure 1}

Conceptual model of the continuum of subclinical atherosclerosis and available measures of vascular health. Inflammatory states downregulate endothelium-dependent nitric oxide production and increase expression of adhesion molecules, leading to the inability to regulate vascular tone, cellular adhesion and thrombosis that characterizes endothelial dysfunction. Subsequent maladaptive cellular changes result in increased arterial stiffness and thickening of the intimal-medial layers. RHI: reactive hyperemia index measures nitric-oxide dependent vasodilatory responses; PWV: pulse wave velocity and analysis approximate arterial stiffness; IMT: intima-media thickness by carotid ultrasound measures structural remodeling, which precedes plaque formation.

\section{Supplementary Files}

This is a list of supplementary files associated with this preprint. Click to download.

- SupplementalTable.docx 